\title{
Continuity of Care Assessment Within a Vertically Integrated Care Management Organization Before and After COPD-Related Exacerbations
}

\author{
Morgan Justice Fuoco' \\ Richard A Mularski (D) \\ Benjamin $\mathrm{Wu}^{2}$ \\ Chad Moretz $\mathbb{D D}^{2}$ \\ Mary Ann McBurnie $\mathbb{D}^{1}$ \\ Richard H Stanford $\mathbb{D}^{2}$ \\ Phillip Crawford (D) ${ }^{\prime}$ \\ Daniel Gratie ${ }^{2}$ \\ Bianca Salas'
}

'Kaiser Permanente Center for Health Research, Science Programs Department, Portland, OR, USA; ${ }^{2}$ GlaxoSmithKline, US Value Evidence and Outcomes, Research Triangle Park, Durham, NC, USA
Correspondence: Morgan Justice Fuoco Kaiser Permanente Washington Health Research Institute, 1730 Minor Ave, Suite I600, Seattle, WA, 9810I, USA

Tel + I 256-508-46/3

Email morgan.j.fuoco@kp.org
Background: There is currently little research describing patient experience and continuity of care immediately prior, during, and following an acute exacerbation of chronic obstructive pulmonary disease (AECOPD). This analysis examined clinical characteristics, chronic obstructive pulmonary disease (COPD)-related medication patterns and outpatient visits before and after an AECOPD.

Methods: This retrospective analysis used electronic health records, medical claims, and pharmacy dispensing data for patients within the Kaiser Permanente Northwest Health System. Patients with $\geq 1$ AECOPD between January 1, 2015 and December 31, 2017 were identified. The most recent AECOPD was considered the index date. An AECOPD was defined as an inpatient hospitalization with a primary diagnosis of COPD, or respiratory failure with a secondary diagnosis of COPD, or an outpatient visit with a primary diagnosis of COPD and dispensing of respiratory-related antibiotics and/or oral corticosteroids \pm 5 days of the visit. Eligible patients were: $\geq 40$ years old; $\geq 2$ encounters within 12 months of each other or $\geq 1$ hospitalization with primary or secondary COPD diagnosis, chronic bronchitis, or emphysema prior to index; and continuously enrolled \pm 90 days relative to index. COPDrelated inhaled maintenance medication, rescue inhalers, oral corticosteroid use, and ambulatory visits were assessed 90-days pre- and post-index.

Results: There were 2829 patients included (mean [standard deviation] age: 69.0 [10.5] years) who had an AECOPD (7\% severe; 93\% moderate). The percentage of patients on inhaled maintenance therapy increased from $60.6 \%$ pre-AECOPD to $68.8 \%$ post-AECOPD and increased from $60.0 \%$ to $87.4 \%$ among patients who experienced a severe AECOPD. COPD-related ambulatory visits increased more than four-fold for primary care and more than doubled for pulmonologist visits in the post-AECOPD period.

Conclusion: The low proportion of patients observed with changes to controller and rescue medication (particularly following a moderate AECOPD), yet higher utilization of COPDrelated ambulatory visits before and after an AECOPD suggests that there is opportunity to improve pharmacotherapy management.

Keywords: COPD, exacerbation, triple therapy, treatment patterns, quality of care

\section{Introduction}

Chronic obstructive pulmonary disease (COPD) is a chronic condition associated with approximately 15.4 million physician visits, 1.5 million emergency department (ED) visits, and 726,000 hospitalizations annually in the United States. ${ }^{1}$ Most patients with COPD are also burdened by comorbidities, ${ }^{2}$ such as cardiovascular disease and depression. ${ }^{3}$ These factors contribute to an estimated annual economic 
burden of $\$ 50$ billion in the United States in 2010. Nearly two thirds of these costs relate directly to COPD diagnosis, treatment, prevention and rehabilitation, which include physician office visits, hospitalizations, home care, and medications. ${ }^{4}$ Indirect costs, relating to reduced productivity or lost days of work due to disease morbidity and mortality, further contribute to the economic burden of COPD. ${ }^{4}$

Many patients suffer from acute exacerbations of COPD (AECOPD), which are defined as an acute worsening of respiratory symptoms that require additional therapy. ${ }^{5}$ AECOPDs can be classified as mild (selfmanaged), moderate (requiring treatment with antibiotics and/or corticosteroids) or severe (requiring hospitalization). ${ }^{6}$ Severe AECOPDs can negatively affect patient prognosis, with mortality rates increasing with the frequency of severe AECOPDs. ${ }^{7}$ Following discharge from hospitalization due to an AECOPD, nearly one fifth of patients are readmitted within 30 days, further adding to the burden on healthcare systems. ${ }^{8}$

The Global Initiative for Chronic Obstructive Lung Disease (GOLD) 2020 report states that the goal for treatment of an AECOPD is to reduce the negative impact of the current AECOPD and to prevent subsequent events. ${ }^{5}$ Short-acting $\beta_{2}$-agonists (SABA) and/or short-acting muscarinic antagonists (SAMA) are recommended as the initial treatment, with maintenance therapy initiated as soon as possible before hospital discharge. ${ }^{5}$ The administration of bronchodilators (anticholinergic agents, $\beta_{2}$-agonists and methylxanthines) within 30 days of a hospital discharge is currently a quality of care measure in the United States. ${ }^{9}$ Mortality statistics show that, for a severe AECOPD, the risk of death is highest in the first week following hospital admission. ${ }^{10}$ This highlights the importance of prompt and effective intervention for patients admitted to hospital with a severe AECOPD. However, adherence to recommended care is often suboptimal, ${ }^{11-14}$ with high levels of under-treatment reported in patients with COPD. ${ }^{12}$

Numerous studies have explored patient characteristics in relation to an AECOPD event or hospital visit/ admission; ${ }^{15-21}$ however, they typically focus on one area of care (eg emergency care) rather than using an integrated care delivery network. There is currently little research describing patient experience and continuity of care immediately prior, during, and following an AECOPD. Examining care for patients before and after an AECOPD within an integrated delivery network could yield information to aid system-level quality improvements. Here we describe the clinical characteristics, COPD-related medication patterns and outpatient medical encounters of patients with COPD, 90 days before and after an AECOPD.

\section{Materials and Methods}

\section{Study Design}

This retrospective, longitudinal analysis used linked electronic health records, medical claims, and pharmacy dispensing data for patients within the Kaiser Permanente Northwest Health System, an integrated health plan and healthcare organization, which serves over 600,000 people in Oregon and Washington, United States. Ethical approval of this study was provided by Kaiser Permanente Northwest Region Institutional Review Board (approval number: 1394778) and a waiver of informed consent was granted.

The study design is shown in Figure 1. We examined data for patients with COPD who experienced a moderate or severe AECOPD between January 1, 2015 and December 31, 2017, with follow-up data from 2018 accessed as needed. The date of the most recently identified AECOPD was considered the index date. The index period was defined as the date of the most recent AECOPD to the day before discharge, which applied only to patients who were hospitalized. Using the electronic health records and claims data at the inpatient and outpatient level, descriptive summaries were obtained for each of the periods of interest: 90 days pre-AECOPD, AECOPD (index), and 90 days post-AECOPD.

\section{Study Population}

Eligible patients fulfilled the following criteria: $\geq 40$ years old at index; $\geq 2$ independent encounter records at least one day apart and within 12 months of each other or $\geq 1$ hospitalization with a primary or secondary diagnosis of COPD (International Classification of Diseases [ICD]-9:491.xx; ICD-10:J44.x), chronic bronchitis (ICD-9: 492.xx; ICD-10: J41.x; J42.x), or emphysema (ICD-9: 496.xx; ICD-10: J43.x) between January 1, 2008 and December 31, 2017; continuously enrolled for 12 months pre-index using Kaiser insurance gap standards (enrolled at least 10 of 12 months); continuously enrolled for \pm 90 days relative to the index date; no AECOPD $<90$ days prior to index. Patients were excluded if, at the time of consent for receipt of 


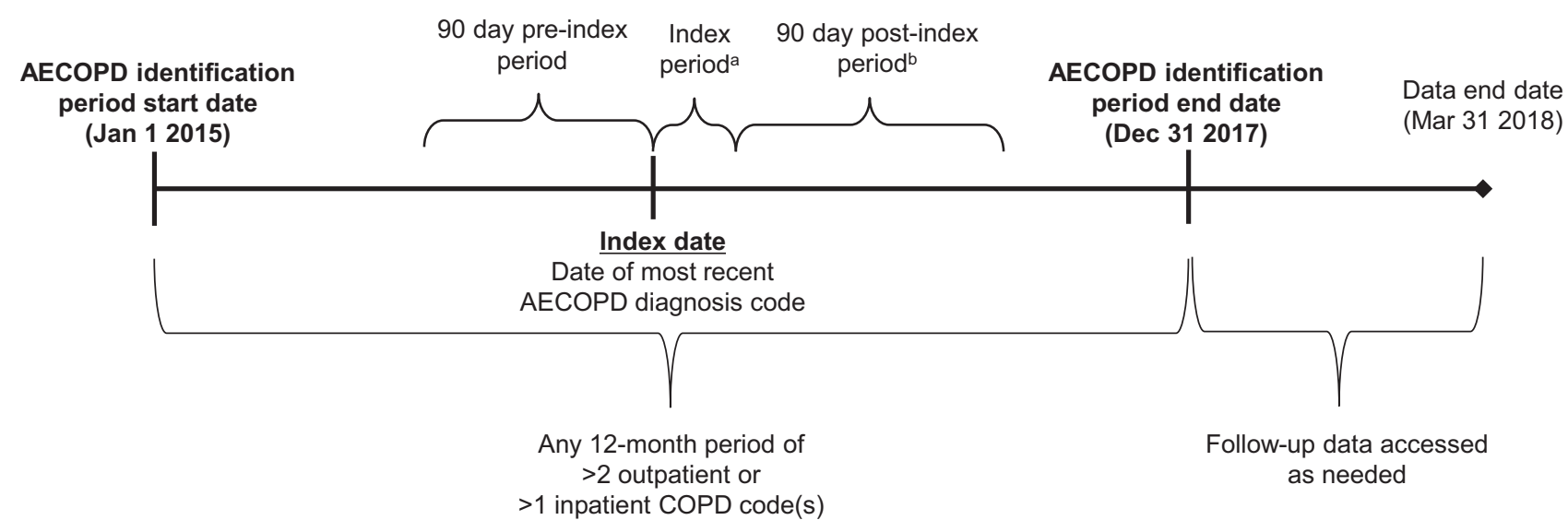

Figure I Study design.

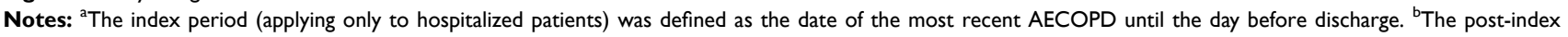
period began I day after hospitalization discharge or I day after outpatient diagnosis code for an AECOPD.

Abbreviations: AECOPD, acute exacerbation of chronic obstructive pulmonary disease; COPD, chronic obstructive pulmonary disease.

medical care within Kaiser Permanente Northwest, they specifically indicated that they did not agree to the use of their health data for research.

A moderate AECOPD was defined as an outpatient visit (ED, physician office, or urgent care) with a primary diagnosis of COPD and dispensing of respiratory-related antibiotics and/or oral corticosteroids \pm 5 days of the visit. A severe AECOPD was defined as: an inpatient hospitalization with a primary diagnosis of COPD; or respiratory failure with a secondary diagnosis of COPD; or an inpatient hospitalization occurring as a result of an ED visit on the same day or the day following the ED visit with a severe acute AECOPD diagnosis. Each patient only contributed one AECOPD to the analysis.

\section{Outcomes}

Variables were gathered using the electronic medical records or dispensing records. COPD-related inhaled maintenance medication, rescue inhalers, oral corticosteroid use, and ambulatory visits were assessed 90 days preand post-index AECOPD. Medication overlap was not considered (ie only the medication dispensing date was recorded, regardless of the days covered by that dispensing), and each prescription dispensing must have occurred within the study period. Any changes in therapy that may have occurred at time of discharge from the hospital, ED, or physician office were assessed in the pre-index, index and post-index periods. Outpatient visits were only considered to be COPD related if a primary COPD diagnostic code was associated with the specific encounter. Missing data were not imputed and are reported as missing.

\section{Results}

\section{Patient Characteristics at Index}

A total of 12,712 patients with COPD were observed during the identification period from January 1, 2015 to December 31, 2017. Of these, 2829 patients (22\%) experienced an AECOPD and were included in the study population. Of the patients with an exacerbation, 190 patients (6.7\%) had a severe AECOPD and 2639 patients $(93.3 \%)$ had a moderate AECOPD.

Patient characteristics at index are shown in Table 1. The mean (standard deviation) age was 69.0 (10.5) years and $52.9 \%$ of the population were female. The mean (standard deviation) duration of COPD was 4.8 (3.2) years. Overall, $26.0 \%$ of patients had concomitant asthma (as defined by encounter diagnosis identified within one year prior to COPD exacerbation), $27.3 \%$ were active smokers, $64.5 \%$ were former smokers and $7.9 \%$ had never smoked.

\section{COPD-Related Treatment at Index}

Table 2 describes the medications dispensed at index. Patients could receive either mono-, dual, or triple therapy, or combinations of rescue and controller inhalers. Oral corticosteroids were the most frequently dispensed drugs during an AECOPD (58.6\% of patients), followed by SABA rescue inhalers (16.1\% of patients). Oral corticosteroids were used slightly more often in patients with a severe AECOPD compared with those who experienced a moderate AECOPD (68.4\% versus 58.9\%, respectively).

In patients who experienced a moderate AECOPD, SABA rescue inhalers were used nearly six times as 
Table I Patient Characteristics at Index

\begin{tabular}{|c|c|c|c|}
\hline & $\begin{array}{l}\text { Overall } \\
(\mathrm{N}=\mathbf{2 8 2 9})\end{array}$ & $\begin{array}{c}\text { Moderate AECOPD } \\
(n=2639)\end{array}$ & $\begin{array}{c}\text { Severe AECOPD } \\
(n=190)\end{array}$ \\
\hline Age (years), mean (SD) & $69.0(10.5)$ & $69.1(10.4)$ & $68.2(11.7)$ \\
\hline \multicolumn{4}{|l|}{ Age group, n (\%) } \\
\hline $40-49$ years & $80(2.8)$ & $70(2.7)$ & $10(5.3)$ \\
\hline $50-64$ years & $926(32.7)$ & 857 (32.5) & $69(36.3)$ \\
\hline$\geq 65$ years & $1823(64.4)$ & $17 \mid 2(64.9)$ & III (58.4) \\
\hline \multicolumn{4}{|l|}{ Gender, n (\%) } \\
\hline Females & $1496(52.9)$ & I $387(52.6)$ & $109(57.4)$ \\
\hline \multicolumn{4}{|l|}{ Race-ethnicity, n (\%) } \\
\hline White NH & 2601 (9I.9) & 2424 (91.9) & 177 (93.2) \\
\hline Black/African American NH & $45(1.6)$ & $41(1.6)$ & $4(2.1)$ \\
\hline Native American/Alaskan Native NH & $13(0.5)$ & $13(0.5)$ & 0 \\
\hline Asian $\mathrm{NH}$ & $21(0.7)$ & $21(0.8)$ & 0 \\
\hline Multi-racial NH & $77(2.7)$ & $73(2.8)$ & $4(2.1)$ \\
\hline Hispanic & $49(1.7)$ & $46(1.7)$ & $3(1.6)$ \\
\hline Other & $8(0.3)$ & $8(0.3)$ & 0 \\
\hline Missing & $15(0.5)$ & $13(0.5)$ & $2(1.1)$ \\
\hline BMI $\left(\mathrm{kg} / \mathrm{m}^{2}\right)$, mean $(\mathrm{SD})$ & $29.64(7.8)$ & $29.57(7.5)$ & $30.51(10.5)$ \\
\hline Weight (kg), mean (SD) & $84.06(24.1)$ & $83.97(23.8)$ & $85.34(28.9)$ \\
\hline Duration of COPD (years), mean (SD) & $4.8(3.2)$ & $4.8(3.2)$ & $4.5(3.4)$ \\
\hline$<$ l year, n (\%) & $237(8.4)$ & $212(8.0)$ & $25(13.2)$ \\
\hline $\mathrm{I}-5$ years, $\mathrm{n}(\%)$ & $1206(42.6)$ & II $24(42.6)$ & $82(43.2)$ \\
\hline$>5$ years, $n(\%)$ & $1386(49.0)$ & $1303(49.4)$ & $83(43.7)$ \\
\hline \multicolumn{4}{|l|}{ Smoking status, $\mathrm{n}(\%)$} \\
\hline Current smoker & $771(27.3)$ & $704(26.7)$ & $67(35.3)$ \\
\hline Former smoker & $1824(64.5)$ & $1720(65.2)$ & $104(54.7)$ \\
\hline Never smoked & $223(7.9)$ & $206(7.8)$ & $17(8.9)$ \\
\hline No information & II (0.4) & $9(0.3)$ & $2(I . I)$ \\
\hline \multicolumn{4}{|l|}{ Vaccination status, n (\%) } \\
\hline Pneumococcal $(\geq 50 \text { years of age })^{a}$ & $2203(77.9)$ & $2065(78.2)$ & $138(72.6)$ \\
\hline Influenza (current season) ${ }^{b}$ & $1597(56.5)$ & $1487(56.3)$ & II $0(57.9)$ \\
\hline Influenza (year prior to index) ${ }^{c}$ & $2042(72.2)$ & $1899(72.0)$ & I $43(75.3)$ \\
\hline Eosinophil count (cells/ $\mu \mathrm{L})-$ highest value ${ }^{d}$ & $281.44(323.1)$ & $279.18(329.4)$ & $307.76(236.6)$ \\
\hline$<150$, mean (SD) & $531(18.8)$ & $497(18.8)$ & $34(17.9)$ \\
\hline I50-300, mean (SD) & $645(22.8)$ & $598(22.7)$ & $47(24.7)$ \\
\hline$>300$, mean $(\mathrm{SD})$ & $518(18.3)$ & $465(17.6)$ & $53(27.9)$ \\
\hline No data, n (\%) & II $35(40.1)$ & $1079(40.9)$ & $56(29.5)$ \\
\hline Eosinophil count - most recent value $(\text { cells } / \mu \mathrm{L})^{\mathrm{e}}$ & $217.09(236.2)$ & $215.59(237.8)$ & $234.55(216.7)$ \\
\hline$<150$, mean $(S D)$ & $741(26.2)$ & $690(26.1)$ & $51(26.8)$ \\
\hline $150-300$, mean $(S D)$ & $592(20.9)$ & $548(20.8)$ & $44(23.2)$ \\
\hline$>300$, mean $(S D)$ & $361(12.8)$ & $322(12.2)$ & $39(20.5)$ \\
\hline No data, n (\%) & 1135 (40.I) & 1079 (40.9) & $56(29.5)$ \\
\hline
\end{tabular}

(Continued) 
Table I (Continued).

\begin{tabular}{|c|c|c|c|}
\hline & $\begin{array}{l}\text { Overall } \\
(\mathrm{N}=\mathbf{2 8 2 9})\end{array}$ & $\begin{array}{c}\text { Moderate AECOPD } \\
(n=2639)\end{array}$ & $\begin{array}{c}\text { Severe AECOPD } \\
(n=190)\end{array}$ \\
\hline Charlson Comorbidity Index score, mean (SD) & $2.94(2.3)$ & $2.92(2.3)$ & $3.19(2.4)$ \\
\hline \multicolumn{4}{|l|}{ Comorbidities, n (\%) } \\
\hline Congestive heart failure $^{f}$ & $5 I I(18.1)$ & $462(17.5)$ & $49(25.8)$ \\
\hline Diabetes $^{f}$ & $776(27.4)$ & $716(27.1)$ & $60(31.6)$ \\
\hline Asthma $^{f}$ & $736(26.0)$ & $689(26.1)$ & $47(24.7)$ \\
\hline Alpha-I antitrypsin deficiency ${ }^{f}$ & $5(0.2)$ & $3(0.1)$ & $2(1.1)$ \\
\hline \multicolumn{4}{|l|}{ Lung function } \\
\hline $\mathrm{FEV}_{\text {। }}(\mathrm{L})$, mean $(\mathrm{SD})$ & $\mathrm{I} .68(0.7)$ & $\mathrm{I} .7(0.7)$ & I.4 (0.6) \\
\hline FVC (L), mean (SD) & $3.03(1.0)$ & $3.05(0.9)$ & $2.76(1.0)$ \\
\hline \multicolumn{4}{|l|}{ Most recent reversibility, n (\%) } \\
\hline Patient has no data & 1257 (44.4) & II 63 (44.I) & $94(49.5)$ \\
\hline No & $970(34.3)$ & $920(34.9)$ & $50(26.3)$ \\
\hline Yes & $602(21.3)$ & $556(21.1)$ & $46(24.2)$ \\
\hline \multicolumn{4}{|l|}{ GOLD Stage, n (\%) } \\
\hline Missing & $758(26.8)$ & $693(26.3)$ & $65(34.2)$ \\
\hline Stage I & $187(6.6)$ & $185(7.0)$ & $2(1.1)$ \\
\hline Stage II & $759(26.8)$ & $717(27.2)$ & $42(22.1)$ \\
\hline Stage III & $345(12.2)$ & $314(1 \mid .9)$ & $31(16.3)$ \\
\hline Stage IV & $61(2.2)$ & $54(2.0)$ & $7(3.7)$ \\
\hline Patient has post $\mathrm{FEV}_{1} / \mathrm{FVC}$, but not $<0.7$ & $219(7.7)$ & $205(7.8)$ & $14(7.4)$ \\
\hline Patient has no post $F E V_{1} / F V C$ or post $F E V_{1}$ predicted & $500(17.7)$ & $47 \mid(17.8)$ & $29(15.3)$ \\
\hline
\end{tabular}

Notes: ${ }^{a}$ One pneumococcal vaccine within last six years prior to index date for AECOPD. ${ }^{b}$ One influenza vaccine during the most recent influenza season (August to March) but not within 14 days of index date. ${ }^{c}$ One influenza vaccine within the past year but not within 14 days of index date. ${ }^{d}$ Highest eosinophil value within the past year

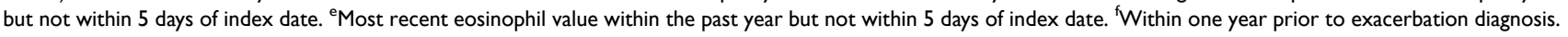
Abbreviations: AECOPD, acute exacerbation of chronic obstructive pulmonary disease; BMI, body mass index; COPD, chronic obstructive pulmonary disease; FEV ${ }_{1}$, forced expiratory volume in I second; FVC, forced vital capacity; GOLD, Global Initiative for Chronic Obstructive Lung Disease; NH, non-Hispanic; SD, standard deviation.

often than a combination of SABA and SAMA $(15.5 \%$ versus $2.8 \%$, respectively). Nebulized SABA rescue therapy was also used more often than nebulized SABA/ SAMA concurrent or combination medications in patients with a moderate AECOPD (5.0\% versus $1.4 \%$, respectively). In patients who experienced a severe AECOPD, SABA rescue inhalers were used three times less often than the combination of SABA and SAMA $(24.7 \%$ versus $77.4 \%$, respectively). However, nebulized SABA rescue therapy was used twice as often as nebulized SABA/ SAMA concurrent or combination medications in patients with a severe AECOPD $(14.1 \%$ versus $15.4 \%$, respectively).

Among patients who experienced a severe AECOPD, $31.6 \%$ were dispensed a long-acting muscarinic antagonist (LAMA), 27.4\% were dispensed an inhaled corticosteroid (ICS)/long-acting $\beta 2$-agonist (LABA) concurrent or combination medication, $17.9 \%$ were dispensed ICS, LABA, LAMA triple therapy and $16.3 \%$ were dispensed an ICS inhaler. However, a maintenance therapy was not dispensed in $31.1 \%$ of patients who experienced a severe AECOPD (Table 3). In comparison, patients who experienced a moderate AECOPD were less likely to be prescribed a controller inhaler; $7.6 \%$ were dispensed a LAMA, 6.3\% were dispensed an ICS/LABA concurrent or combination medication, $1.8 \%$ were dispensed ICS, LABA, LAMA triple therapy, and $4.6 \%$ were dispensed an ICS inhaler.

\section{COPD-Related Treatment Pre- and Post-AECOPD}

The use of controller and rescue COPD medication throughout the study period is shown in Table 3. Prior to an AECOPD, patients showed similar patterns of controller medication use regardless of whether they subsequently experienced a moderate or severe AECOPD. Overall, the percentage of patients using inhaled maintenance therapy 
Table 2 COPD-Related Therapy Dispensed in the AECOPD Group at Index ${ }^{\mathrm{a}, \mathrm{b}}$

\begin{tabular}{|c|c|c|c|}
\hline & $\begin{array}{l}\text { Overall } \\
\mathbf{N}(\%)\end{array}$ & $\begin{array}{c}\text { Moderate AECOPD } \\
\text { n (\%) }\end{array}$ & $\begin{array}{c}\text { Severe AECOPD } \\
\text { n (\%) }\end{array}$ \\
\hline Overall, $\mathrm{N}$ & 2829 & 2639 & 190 \\
\hline \multicolumn{4}{|l|}{ Controller inhalers } \\
\hline LAMA & $260(9.2)$ & $200(7.6)$ & $60(31.6)$ \\
\hline LABA & $7(0.2)$ & $6(0.2)$ & $\mathrm{I}(0.5)$ \\
\hline LAMA+LABA or LAMA/LABA ${ }^{c}$ & 0 & 0 & 0 \\
\hline$I C S+L A B A$ or ICS/LABA ${ }^{c}$ & $218(7.7)$ & $166(6.3)$ & $52(27.4)$ \\
\hline$I C S+L A M A+L A B A$ or ICS/LAMA/LABA ${ }^{c}$ & $81(2.9)$ & $47(1.8)$ & $34(17.9)$ \\
\hline ICS & $153(5.4)$ & $122(4.6)$ & $31(16.3)$ \\
\hline \multicolumn{4}{|l|}{ Rescue inhalers } \\
\hline SABA & $456(16.1)$ & $409(15.5)$ & $47(24.7)$ \\
\hline SAMA & $23(0.8)$ & $15(0.6)$ & $8(4.2)$ \\
\hline SABA+SAMA or SABA/SAMA ${ }^{c}$ & $220(7.8)$ & $73(2.8)$ & 147 (77.4) \\
\hline Oral corticosteroid & $1657(58.6)$ & $1527(57.9)$ & $130(68.4)$ \\
\hline \multicolumn{4}{|l|}{ Oral COPD therapy } \\
\hline Methylxanthines & $3(0.1)$ & I $(0.0)$ & $2(1.1)$ \\
\hline PDE-4 inhibitors & $\mathrm{I}(0.0)$ & 0 & $\mathrm{I}(0.5)$ \\
\hline \multicolumn{4}{|l|}{ Nebulized controller therapy } \\
\hline LAMA & 0 & 0 & 0 \\
\hline LABA & 0 & 0 & 0 \\
\hline$L A M A+L A B A$ or $L A M A / L A B A^{c}$ & 0 & 0 & 0 \\
\hline$I C S+L A B A$ or $I C S / L A B A^{c}$ & 0 & 0 & 0 \\
\hline ICS+LAMA+LABA or ICS/LAMA/LABA ${ }^{c}$ & 0 & 0 & 0 \\
\hline ICS & $2(0.1)$ & 0 & $2(1.1)$ \\
\hline \multicolumn{4}{|l|}{ Nebulized rescue therapy } \\
\hline SABA & $211(7.5)$ & $133(5.0)$ & $78(4 I .1)$ \\
\hline SAMA & $27(1.0)$ & $13(0.5)$ & $14(7.4)$ \\
\hline SABA+SAMA or SABA/SAMA ${ }^{c}$ & $67(2.4)$ & $38(1.4)$ & $29(15.3)$ \\
\hline
\end{tabular}

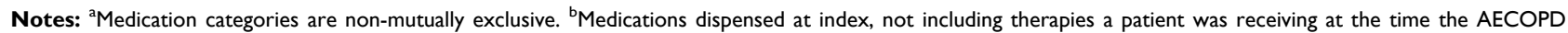
occurred. ${ }^{\circ}$ Classifications with a slash (/) indicate combination medications, a plus (+) indicates simultaneous dispensings.

Abbreviations: AECOPD, acute exacerbation of chronic obstructive pulmonary disease; COPD, chronic obstructive pulmonary disease; ICS, inhaled corticosteroid; LABA, long-acting $\beta_{2}$-agonist; LAMA, long-acting muscarinic antagonist; PDE-4, phosphodiesterase-4; SABA, short-acting $\beta_{2}$-agonist; SAMA, short-acting muscarinic antagonist.

increased from $60.6 \%$ pre-AECOPD to $68.8 \%$ postAECOPD. Of note, there was a large increase in the proportion of patients receiving inhaled maintenance therapy postAECOPD among patients who experienced a severe AECOPD: $60.0 \%$ pre-AECOPD versus $87.4 \%$ postAECOPD. In contrast, among patients with a moderate AECOPD, inhaled maintenance therapy use increased from $60.7 \%$ pre-AECOPD to $67.4 \%$ post-AECOPD.

The use of ICS therapy alone was similar in the preand post-index periods (7.3\% versus $7.5 \%$, respectively). The use of ICS/LABA+LAMA and ICS+LAMA approximately doubled following an AECOPD event for patients who experienced a severe AECOPD (22.6\% versus $42.1 \%$ and $5.3 \%$ versus $10.5 \%$, respectively). The increase was much smaller for patients who experienced a moderate AECOPD (19.9\% versus $24.7 \%$ and $5.1 \%$ versus $6.6 \%$, respectively). Overall, the use of multiple-inhaler triple therapy (ICS+LAMA+LABA) increased from $20.1 \%$ to $25.9 \%$ between the pre-index to the post-index period. The use of rescue inhalers did not markedly change between the pre- and post-index periods $(52.4 \%$ versus $53.0 \%$, respectively).

\section{Healthcare Utilization}

COPD-related healthcare utilization is shown in Table 4. The percentage of patients with at least one COPD-related 


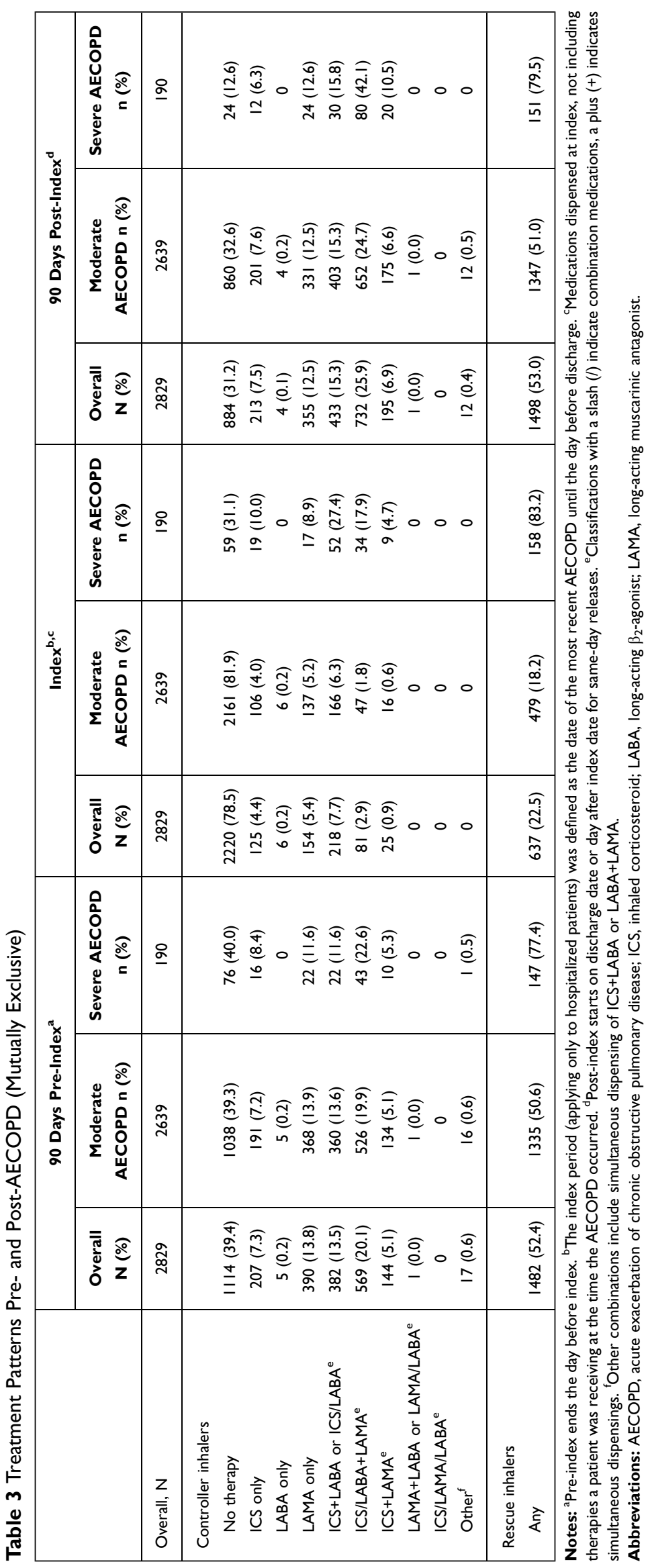




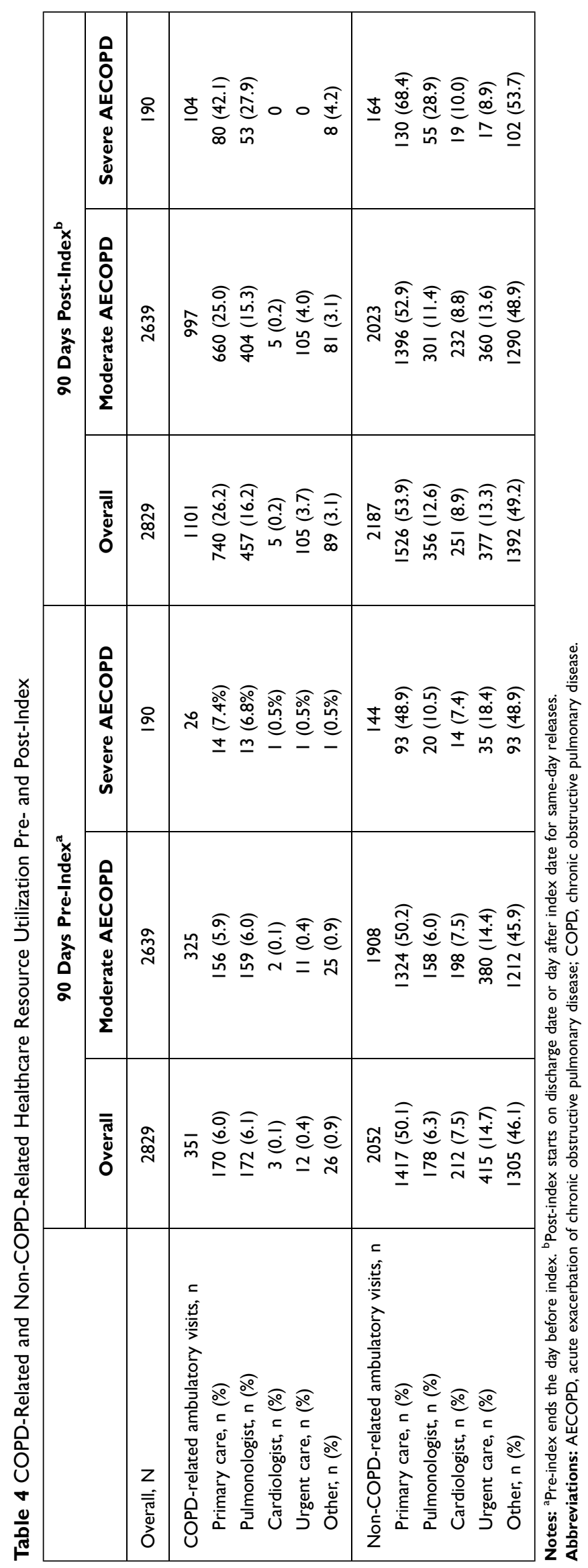


ambulatory visit increased more than four-fold for primary care from the pre-AECOPD to the post-AECOPD period $(6.0 \%$ versus $26.2 \%$ of patients, respectively). The increase in primary care visits was greater among patients who experienced a severe AECOPD (7.4\% pre-AECOPD versus $42.1 \%$ post-AECOPD) compared with those who experienced a moderate AECOPD (5.9\% pre-AECOPD versus $25.0 \%$ post-AECOPD, respectively).

The proportion of patients with COPD-related pulmonologist visits also increased from the pre-AECOPD period to the post-AECOPD period $(6.1 \%$ versus $15.3 \%$, respectively). This increase was also greater for patients who experienced a severe AECOPD (6.8\% pre-AECOPD versus $27.9 \%$ post-AECOPD) compared with those who experienced a moderate AECOPD (6.0\% pre-AECOPD versus $15.3 \%$ post-AECOPD). In the post-AECOPD period, $30(1.1 \%)$ patients had a COPD-related hospitalization.

Non-COPD-related healthcare utilization is also shown in Table 4. Overall, $53.9 \%$ of patients made at least one non-COPD-related primary care visit and $12.6 \%$ of patients made at least one pulmonologist visit in the postAECOPD period. The proportion of patients with a severe AECOPD making a primary care visit increased from $48.9 \%$ pre-AECOPD to $68.4 \%$ post-AECOPD. Among patients who had a moderate AECOPD, the proportion of patients who had a primary care visit increased from $50.2 \%$ pre-AECOPD to $52.9 \%$ post-AECOPD. In total, $104(3.6 \%)$ patients had a non-COPD-related hospitalization in the pre-AECOPD period compared with 122 patients $(4.3 \%)$ in the post-AECOPD period.

\section{Discussion}

This study aimed to describe the COPD-related medication patterns and outpatient encounters 90 days before and after an AECOPD. Most patients identified in our study experienced a moderate AECOPD at index and, consistent with the GOLD 2020 report for patients with an AECOPD, ${ }^{5}$ the majority of patients included in the study received systemic corticosteroids during AECOPD events. However, observed dispensing rates suggest that there is room for improvement in care delivery and pharmacotherapy. For example, a high proportion of patients with COPD were not on an inhaled maintenance therapy prior to, and after, an AECOPD. Although the percentage of patients not receiving any COPD therapy decreased slightly in the post-AECOPD period compared with the pre-AECOPD period, almost one third of patients remained without therapy following an AECOPD. Although the patients who experienced a severe AECOPD were more likely to receive maintenance therapy following their AECOPD, $12.6 \%$ of these patients remained without therapy in the post-AECOPD period.

We also observed an increase in the proportion of patients with COPD-related ambulatory visits in the postAECOPD period compared with the pre-AECOPD period, particularly among patients who had experienced a severe AECOPD. This pattern of increased visits in the postAECOPD period could suggest either a lack of COPD control following the AECOPD, or increased clinical attention to ensure post-AECOPD care optimization. Coupled with the increased use of inhaled maintenance therapy following a severe AECOPD, the results suggest the latter, that severe AECOPD drives a shift in the pattern of care and pharmacological treatment provided for COPD patients.

Treatment guidelines recommend SABA/SAMA therapy during an AECOPD followed by maintenance therapy as soon as possible before discharge. ${ }^{5}$ Our data suggest that the utilization of short-acting inhaled therapy could possibly be improved, as less than a quarter of patients received rescue inhalers at index. The proportion of patients receiving SABA/SAMA rescue medication (both inhalers and nebulized therapy) at index was higher among patients who had a severe AECOPD. In addition, the increased use of controller medications observed in the post-AECOPD period is generally consistent with COPD guideline recommendations. However, guideline adherence assessment at the individual patient level was beyond the scope and intent of this analysis.

Our findings broadly align with previous literature examining lack of optimized maintenance treatment despite a history of AECOPD. ${ }^{11,12,22}$ Diette et al found that $36 \%$ of patients with COPD were not using inhaled maintenance therapy despite a history of exacerbation, ${ }^{11}$ which was similar to our finding for patients in this category $(31.2 \%)$. In their retrospective cohort study, Price et al observed that $43 \%$ of patients with COPD were not prescribed the guideline-recommended treatment in the previous year, ${ }^{23}$ while Make et al reported that, among patients hospitalized for COPD, $40.2 \%$ of patients with commercial insurance and $29.9 \%$ of patients with Medicare remained untreated 45 days following hospitalization. ${ }^{12}$ In contrast, we describe a lower proportion of patients untreated 90 days after a severe AECOPD $(12.6 \%)$, despite using a broader definition for 
bronchodilators. $^{12}$ It is possible that this difference could be due to a longer post-index period in the present study, which would suggest that a proportion of patients receive therapy between 45 and 90 days post-AECOPD. Alternatively, the lower proportion of untreated patients in our study could reflect standardized care procedures within the Kaiser Permanente Northwest Health System. The lower percentage of untreated patients in our analysis could also potentially be explained by a difference of over 10 years in the observation window and improvements in standards of COPD care over this period.

In a retrospective analysis of inpatient data collected in an administrative database in the United States, the majority of patients with COPD received treatment for an AECOPD in line with GOLD guideline recommendations; $95 \%, 81 \%$, and $92 \%$ of patients received SABAs, systemic corticosteroids, and antibiotics, respectively. ${ }^{22}$ However, long-acting bronchodilator maintenance therapy had been initiated in only half of patients $(52.2 \%)$ before discharge. In contrast, the present study found that only $83.2 \%$ of patients with a severe AECOPD received rescue therapy during the AECOPD. However, we identified a higher proportion of patients with a severe AECOPD receiving maintenance therapy during the exacerbation (68.9\%) compared with the study by Amin et al. ${ }^{22}$ Of note, Amin et al found that patients who did receive maintenance therapy had significantly different characteristics compared with those who did not; for example, patients treated with long-acting bronchodilators had more primary COPD diagnoses, more prior all-cause hospitalizations, longer lengths of hospital stay, fewer comorbidities, and greater spirometry use. ${ }^{22}$ This information could be used to identify patient populations with an unmet need for optimized therapy.

We observed that the largest difference in pharmacotherapy management after experiencing an AECOPD was an increased use of ICS+LAMA+LABA triple therapy. Recent clinical trials suggest that ICS+LAMA + LABA triple therapy can improve some post-AECOPD outcomes. $^{24-27}$ Lipson et al demonstrated that in symptomatic patients with a history of exacerbations, fluticasone furoate, umeclidinium and vilanterol (FF/UMEC/VI) significantly reduced the annual rate of moderate/severe AECOPD compared with dual therapies (FF/VI and UMEC/VI) and significantly reduced COPD-related hospitalizations compared with UMEC/VI. ${ }^{25}$ Additionally, Bogart et al demonstrated that initiation of triple therapy within 30 days of an AECOPD was associated with reduced AECOPD rates and reduced COPD-related healthcare costs compared with delayed therapy. ${ }^{26}$ The recently approved triple therapy comprising budesonide/glycopyrrolate/formoterol fumarate (BUD/GLY/FOR) was shown to significantly reduce rates of moderate-to-severe exacerbations compared with dual therapies BUD/FOR and GLY/FOR. ${ }^{27}$ In our study, there was a slight increase in the proportion of patients with a moderate AECOPD who received ICS+LAMA+LABA triple therapy, from 19.9\% of patients pre-index to $24.7 \%$ post-index. Meanwhile, the proportion of patients with a severe AECOPD who received ICS $+\mathrm{LAMA}+\mathrm{LABA}$ nearly doubled from the pre-index to post-index periods, from $22.6 \%$ to $42.1 \%$. Further research is needed to confirm if the potential benefits of triple therapy post-AECOPD are recognized in patients who experience a moderate AECOPD, as well as in those who experience a severe AECOPD. The observed under-treatment and low augmentation to triple therapy, or any inhaled maintenance therapy, following an AECOPD may be an important contributing factor to the increased number of patients with COPD-related outpatient visits in the post-index period of our study.

Our study has a number of strengths that should be mentioned. First, data were obtained from a population of patients served by an integrated delivery network, which captures most aspects of care provided by the healthcare system. Second, the data included clinical metrics from electronic health records that are not found in typical claims databases, for example additional diagnosis data, laboratory test results, lung function data, and dispensed medications. Additionally, our analysis reported medication dispensing, which is expected to be a closer measure of medication use compared with prescriptions. However, the number of dispensed medications is still likely to be higher than actual patient adherence. It is unlikely that COPD medication use was underestimated in our analysis, as sample medications are not provided to patients treated in the Kaiser Permanente Northwest Health System.

The study also has some limitations that should be taken into consideration. As data in the electronic medical records were not originally collected for research purposes, there is potential for data that does not reliably reflect actual patient care. Additionally, only patients who had consistent use of the health system for 10 out of the 12 months prior to their AECOPD were eligible and included in the analysis. Therefore, our findings may not apply to patients who do not routinely access the healthcare system, or who regularly move between systems. 
Furthermore, patients with no or low levels of insurance coverage are not well represented in the Kaiser Permanente Northwest Health System. Some important aspects of COPD care were not captured, such as the extent of inhaler device training, smoking cessation counselling or pulmonary rehabilitation after an AECOPD. Additionally, patient adherence to the prescribed therapy could not be verified. The number of post-AECOPD pharmacy dispensings may be higher than actual medication use, depending on the amount of medication patients were dispensed at discharge or had prior to the AECOPD. As the analysis was descriptive, it did not formally test any hypotheses nor draw any causal conclusions, and treatment patterns were not evaluated by patient characteristics (or any other characteristics). A further limitation is that the patient population was homogeneous with respect to geographic region and race/ethnicity groups and so may not apply to other regions or patient demographics. In addition, most COPD patients included in the analysis did not have confirmatory spirometry of their COPD diagnosis, akin to the longstanding $<50 \%$ rate seen in the Healthcare Effectiveness Data and Information Set measures; this limitation therefore applies to many real-world data analyses and includes the possibility of misdiagnosis or over- or under- diagnosis in the population. Finally, as the study sought to evaluate medication patterns following an AECOPD and thus required 90-day continuous enrollment following the AECOPD, patients who died in this period were not included, introducing a selection bias against those individuals.

\section{Conclusions}

Within this vertically integrated care management organization, appropriate increases in therapy aligned with clinical practice guidelines were observed, yet still a third of patients who experienced an AECOPD did not receive maintenance therapy post-exacerbation. These results highlight a continuing need to focus on pharmacotherapy management in patients following an AECOPD, to improve physicians' awareness of COPD treatment recommendations, and to identify factors associated with physician prescribing and patient adherence. This information could be used to inform system-level quality improvements and subsequent population health research. Improving adherence to treatment guidelines post-AECOPD may help to reduce healthcare resource utilization.

\section{Abbreviations}

AECOPD, acute exacerbation of chronic obstructive pulmonary disease; BMI, body mass index; COPD, chronic obstructive pulmonary disease; ED, emergency department; FEV1, forced expiratory volume in 1 second; FF, fluticasone furoate; FVC, forced vital capacity; GOLD, Global Initiative for Chronic Obstructive Lung Disease; ICD, International Classification of Diseases; ICS, inhaled corticosteroid; LABA, long-acting $\beta_{2}$-agonist; LAMA, long-acting muscarinic antagonist; $\mathrm{NH}$, non-Hispanic; PDE-4, phosphodiesterase-4; SABA, short-acting $\beta_{2}$-agonist; SAMA, short-acting muscarinic antagonist; SD, standard deviation; UMEC, umeclidinium; VI, vilanterol.

\section{Data Sharing Statement}

The data analyzed in this manuscript are contained in a database owned by Kaiser Permanente Northwest and contain proprietary elements. Therefore, it cannot be broadly disclosed or made publicly available at this time.

\section{Ethics Approval and Informed Consent}

Ethical approval of this study was provided by Kaiser Permanente - Northwest Region Institutional Review Board (approval number: 1394778) and a waiver of informed consent was granted on the basis that there was minimal risk to the patient associated with the planned data collection. The study was conducted in accordance with the Declaration of Helsinki.

\section{Patient Confidentiality}

All patient records included in this analysis were anonymized.

\section{Acknowledgments}

Editorial support (in the form of writing assistance, collating author comments, assembling tables/figures, grammatical editing and referencing) was provided by Bonnie Nicholson, $\mathrm{PhD}$, of Gardiner-Caldwell Communications (Macclesfield, UK) and Fiona Goodwin of Aura, a division of Spirit Medical Communications Group Limited (Manchester, UK), and was funded by GlaxoSmithKline. Trademarks are the property of their respective owners.

These data have previously been submitted/presented in abstract/poster form to the American Thoracic Society 2020 International Conference (Mularski R et al. Continuity of Care Assessment Within a Vertically 
Integrated Care Management Organization Before and After COPD-Related Exacerbations. ATS 2020). This abstract was published in the American Journal of Respiratory and Critical Care Medicine. 2020;201:A4811.

\section{Author Contributions}

All authors made a significant contribution to the work reported, whether that was in the conception, study design, execution, acquisition of data, analysis and interpretation, or in all these areas; took part in drafting, revising, or critically reviewing the manuscript; gave final approval of the version to be published; have agreed on the journal to which the article has been submitted; and agree to be accountable for all aspects of the work.

\section{Funding}

This study was funded by GlaxoSmithKline (study number HO-18-18806). The sponsor was involved in study conception and design, data interpretation, and the decision to submit the article for publication. The sponsor was also given the opportunity to review the manuscript for medical and scientific accuracy as well as intellectual property considerations.

\section{Disclosure}

The authors declare the following conflicts of interest during the last three years in relation to this article: CM was an employee of GlaxoSmithKline at the time the study was conducted and owns stocks in GlaxoSmithKline. CM is currently affiliated with Avalere Health, Health Economics and Advanced Analytics, Washington, DC, USA. BW, RHS, and DG were employees of GlaxoSmithKline at the time the study was conducted. RHS and DG are currently affiliated with AESARA Inc, Chapel Hill, NC, USA. MJF, RAM, MAM, PC, and BS received a collaborative grant from GlaxoSmithKline to conduct this analysis. MJF is currently affiliated with Kaiser Permanente Washington Health Research Institute, Seattle, WA, USA. RAM has served on an advisory board for GlaxoSmithKline. The authors report no other conflicts of interest in this work.

\section{References}

1. May SM, Li JTC. Burden of chronic obstructive pulmonary disease: healthcare costs and beyond. Allergy Asthma Proc. 2015;36(1):4-10. doi:10.2500/aap.2015.36.3812

2. Schnell K, Weiss CO, Lee T, et al. The prevalence of clinicallyrelevant comorbid conditions in patients with physician-diagnosed COPD: a Cross-Sectional Study using data from NHANES 19992008. BMC Pulm Med. 2012;12(1):26. doi:10.1186/1471-2466-12-26
3. Franssen FME, Rochester CL. Comorbidities in patients with COPD and pulmonary rehabilitation: do they matter? Eur Respir Rev. 2014;23(131):131. doi:10.1183/09059180.00007613

4. Guarascio AJ, Ray SM, Finch CK, Self TH. The clinical and economic burden of chronic obstructive pulmonary disease in the USA. Clinicoecon Outcomes Res. 2013;5:235-245. doi:10.2147/CEOR. S34321

5. Global Initiative for Chronic Obstructive Pulmonary Disease (GOLD). Report; 2020. Available from: https://goldcopd.org/. Accessed August, 2020.

6. Pavord ID, Jones PW, Burgel P-R, Rabe KF. Exacerbations of COPD. Int J Chron Obstruct Pulmon Dis. 2016;11(Spec Iss):21-30. doi:10.2147/COPD.S85978

7. Soler-Cataluña JJ, Martínez-García MA, Román Sánchez P, Salcedo E, Navarro M, Ochando R. Severe acute exacerbations and mortality in patients with chronic obstructive pulmonary disease. Thorax. 2005;60(11):925-931. doi:10.1136/thx.2005.040527

8. Jacobs DM, Noyes K, Zhao J, et al. Early hospital readmissions after an acute exacerbation of chronic obstructive pulmonary disease in the nationwide readmissions database. Ann Am Thorac Soc. 2018;15 (7):837-845. doi:10.1513/AnnalsATS.201712-913OC

9. National Committee for Quality Assurance. Pharmacotherapy management of COPD exacerbation. Available from http://passporthealth plan.com/wp-content/uploads/2015/10/PCE-Pharmacotherapy-

Management-of-COPD-Exacerbation.pdf. Accessed August, 2020.

10. Suissa S, Dell'Aniello S, Ernst P. Long-term natural history of chronic obstructive pulmonary disease: severe exacerbations and mortality. Thorax. 2012;67(11):957-963. doi:10.1136/thoraxjnl2011-201518

11. Diette GB, Dalal AA, D'Souza AO, Lunacsek OE, Nagar SP. Treatment patterns of chronic obstructive pulmonary disease in employed adults in the United States. Int J Chron Obstruct Pulmon Dis. 2015;10:415-422. doi:10.2147/COPD.S75034

12. Make B, Dutro MP, Paulose-Ram R, Marton JP, Mapel DW. Undertreatment of COPD: a retrospective analysis of US managed care and Medicare patients. Int J Chron Obstruct Pulmon Dis. 2012;7:1-9. doi:10.2147/COPD.S27032

13. Mularski RA, Asch SM, Shrank WH, et al. The quality of obstructive lung disease care for adults in the United States as measured by adherence to recommended processes. Chest. 2006;130 (6):1844-1850. doi:10.1378/chest.130.6.1844

14. Chan KP, Ko FW, Chan HS, et al. Adherence to a COPD treatment guideline among patients in Hong Kong. Int $J$ Chron Obstruct Pulmon Dis. 2017;12:3371-3379. doi:10.2147/COPD.S147070

15. Genao L, Durheim MT, Mi X, Todd JL, Whitson HE, Curtis LH. Early and long-term outcomes of older adults after acute care encounters for chronic obstructive pulmonary disease exacerbation. Ann Am Thorac Soc. 2015;12(12):1805-1812. doi:10.1513/ AnnalsATS.201504-250OC

16. Hasegawa K, Tsugawa Y, Tsai C-L, Brown DF, Camargo CA Jr. Frequent utilization of the emergency department for acute exacerbation of chronic obstructive pulmonary disease. Respir Res. 2014;15 (1):40. doi:10.1186/1465-9921-15-40

17. Kerkhof M, Freeman D, Jones R, Chisholm A, Price DB, Respiratory Effectiveness G. Predicting frequent COPD exacerbations using primary care data. Int $J$ Chron Obstruct Pulmon Dis. 2015;10:2439-2450. doi:10.2147/COPD.S94259

18. Kumbhare SD, Beiko T, Wilcox SR, Strange C. Characteristics of COPD patients using United States emergency care or hospitalization. Chronic Obstr Pulm Dis. 2016;3(2):539-548. doi:10.15326/jcopdf.3.2.2015.0155

19. Perera PN, Armstrong EP, Sherrill DL, Skrepnek GH. Acute exacerbations of COPD in the United States: inpatient burden and predictors of costs and mortality. COPD. 2012;9(2):131-141. doi:10.3109/ 15412555.2011.650239 
20. Rezaee ME, Ward CE, Nuanez B, Rezaee DA, Ditkoff J, Halalau A Examining 30-day COPD readmissions through the emergency department. Int J Chron Obstruct Pulmon Dis. 2017;13:109-120. doi:10.2147/COPD.S147796

21. Tran M, Xiang P, Rascati KL, et al. Predictors of appropriate pharmacotherapy management of COPD exacerbations and impact on 6-month readmission. J Manag Care Spec Pharm. 2016;22 (10):1186-1193. doi:10.18553/jmcp.2016.22.10.1186

22. Amin AN, Bollu V, Stensland MD, Netzer L, Ganapathy V. Treatment patterns for patients hospitalized with chronic obstructive pulmonary disease. Am J Health Syst Pharm. 2018;75(6):359-366. doi:10.2146/ajhp160979

23. Price L, Billups SJ, Rice MA, Hartsfield C. Investigation of barriers to clinical practice guideline-recommended pharmacotherapy in the treatment of COPD. Pharm Pract (Granada). 2007;5(2):74-77. doi:10.4321/s1886-36552007000200004
24. Lipson DA, Barnacle H, Birk R, et al. FULFIL Trial: once-daily triple therapy for patients with chronic obstructive pulmonary disease. Am $J$ Respir Crit Care Med. 2017;196(4):438-446. doi:10.1164/ rccm.201703-0449OC

25. Lipson DA, Barnhart F, Brealey N, et al. Once-daily single-inhaler triple versus dual therapy in patients with COPD. $N$ Engl J Med. 2018;378(18):1671-1680. doi:10.1056/NEJMoa1713901

26. Bogart M, Glassberg MB, Reinsch T, Stanford RH. Impact of prompt versus delayed initiation of triple therapy post COPD exacerbation in a US-managed care setting. Respir Med. 2018;145:138-144. doi:10.1016/j.rmed.2018.10.01

27. Rabe KF, Martinez FJ, Ferguson GT, et al. Triple inhaled therapy at two glucocorticoid doses in moderate-to-very-severe COPD. $N$ Engl $J$ Med. 2020;383(1):35-48. doi:10.1056/NEJMoa1916046

\section{Publish your work in this journal}

The International Journal of COPD is an international, peer-reviewed journal of therapeutics and pharmacology focusing on concise rapid reporting of clinical studies and reviews in COPD. Special focus is given to the pathophysiological processes underlying the disease, intervention programs, patient focused education, and self management protocols. This journal is indexed on PubMed Central, MedLine and CAS. The manuscript management system is completely online and includes a very quick and fair peer-review system, which is all easy to use. Visit http://www.dovepress.com/testimonials.php to read real quotes from published authors. 